Pacific

Journal of

Mathematics

\title{
TRIGONAL MINIMAL SURFACES IN FLAT TORI
}

TOSHIHIRO SHODA

Volume $232 \quad$ No. 2

October 2007 


\title{
TRIGONAL MINIMAL SURFACES IN FLAT TORI
}

\author{
TOSHIHIRO SHODA
}

\begin{abstract}
We study trigonal minimal surfaces in flat tori. showing first a topological obstruction similar to that of hyperelliptic minimal surfaces: the genus of a trigonal minimal surface in a 3-dimensional flat torus must be $1(\bmod 3)$. Next we construct an explicit example of a trigonal minimal surface in a 4-dimensional flat torus. This surface satisfies good properties and is theoretically distinct from earlier examples.
\end{abstract}

\section{Introduction}

Let $f: M_{g} \rightarrow \mathbb{R}^{n} / \Lambda$ be a minimal immersion of a compact surface of genus $g$ into an $n$-dimensional flat torus, and suppose that $f$ does not lie in any subtorus of $\mathbb{R}^{n} / \Lambda$. (Clearly, $f$ can be replaced by an $n$-periodic minimal immersion from some covering space of $M_{g}$ into $\mathbb{R}^{n}$.) The conformal structure induced by the isothermal coordinates makes $M_{g}$ a Riemann surface and $f$ is called a conformal minimal immersion.

Theorem 1.1 (Generalized Weierstrass Representation [Meeks 1990, p. 884]). Let $f: M_{g} \rightarrow \mathbb{R}^{n} / \Lambda$ be a conformal minimal immersion. After a translation, $f$ can be represented by

$$
f(p)=\operatorname{Re} \int_{p_{0}}^{p}\left(\omega_{1}, \omega_{2}, \ldots, \omega_{n}\right)^{T} \bmod \Lambda,
$$

where $p_{0}$ is a fixed point in $M_{g}$, superscript $T$ means transpose, and $\omega_{1}, \omega_{2}, \ldots$, $\omega_{n}$ are holomorphic differentials on $M_{g}$ such that

(1) $\omega_{1}, \omega_{2}, \ldots, \omega_{n}$ have no common zeros,

(2) $\sum_{k=1}^{n} \omega_{k}^{2}=0$, and

(3) $\left\{\operatorname{Re} \int_{\gamma}\left(\omega_{1}, \omega_{2}, \ldots, \omega_{n}\right)^{T} \mid \gamma \in H_{1}\left(M_{g}, \mathbb{Z}\right)\right\}$ is a sublattice of $\Lambda$.

Conversely, if $\omega_{1}, \omega_{2}, \ldots, \omega_{n}$ are holomorphic differentials satisfying (1), (2), and (3), then $f$, defined as above, is a conformal minimal immersion.

MSC2000: 53A10, 53C42.

Keywords: minimal surfaces, flat tori, trigonal curves. 
Condition (3) is called the period condition and guarantees the well-definedness of the path integral.

Using these notations, we define the associate surface $f_{\theta}$ :

$$
f_{\theta}(p):=\operatorname{Re} \int_{p_{0}}^{p} e^{i \theta}\left(\omega_{1}, \omega_{2}, \ldots, \omega_{n}\right)^{T} .
$$

The associate surface $f_{\pi / 2}$ is called the conjugate surface. We note that $f_{\theta}$ may not be well defined for any torus, even though $f=f_{0}$ is well defined on $M_{g}$.

Recall that the Gauss map $G$ is the holomorphic map from $M_{g}$ to the quadric $Q_{n-2}:=\left\{\left[w_{1}, w_{2}, \ldots, w_{n}\right] \in \mathbb{C P}^{n-1} \mid \sum_{k=1}^{n}\left(w_{k}\right)^{2}=0\right\}$ given by

$$
\begin{aligned}
G: M_{g} & \rightarrow Q_{n-2} \subset \mathbb{C P}^{n-1}, \\
p & \longmapsto\left[\omega_{1}(p), \omega_{2}(p), \ldots, \omega_{n}(p)\right] .
\end{aligned}
$$

One of the beautiful classical theorems on compact Riemann surfaces states that every compact Riemann surface of positive genus is holomorphically embedded in the Jacobian by the Abel-Jacobi map: take a basis $\left\{\eta_{1}, \eta_{2}, \ldots, \eta_{g}\right\}$ of the space of holomorphic differentials of $M_{g}$, and consider

$$
\Lambda_{\eta}=\left\{\operatorname{Re} \int_{\gamma}\left(\eta_{1}, \ldots, \eta_{g},-i \eta_{1}, \ldots,-i \eta_{g}\right)^{T} \mid \gamma \in H_{1}\left(M_{g}, \mathbb{Z}\right)\right\} .
$$

The Jacobian $\operatorname{Jac}\left(M_{g}\right)$ is the complex torus represented by $\mathbb{C}^{g} / \Lambda_{\eta}$ and the holomorphic embedding $j: M_{g} \rightarrow \operatorname{Jac}\left(M_{g}\right)$ defined by

$$
j(p)=\operatorname{Re} \int_{p_{0}}^{p}\left(\eta_{1}, \ldots, \eta_{g},-i \eta_{1}, \ldots,-i \eta_{g}\right)^{T}
$$

is the Abel-Jacobi map. The Jacobian satisfies the following universal property:

Theorem 1.2 [Nagano and Smyth 1980, p. 5]. Given a conformal minimal immersion $f: M_{g} \rightarrow \mathbb{R}^{n} / \Lambda$, and assuming without loss of generality that $f\left(p_{0}\right)=0$, there exists a real homomorphism $h$ from $\operatorname{Jac}\left(M_{g}\right)$ to $\mathbb{R}^{n} / \Lambda$ so that $f=h \circ j$ :

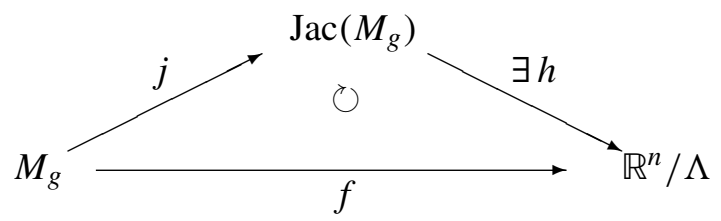

The Abel-Jacobi map plays an important role in the theory of algebraic curves (Torelli's Theorem, the Schottky problem, etc.) and so, by Theorem 1.2, it is useful to study minimal surfaces from the point of view of the theory of algebraic curves. In fact, Ejiri [2002] translated the Schottky problem into a differential geometric situation and studied the moduli space of compact minimal surfaces in flat tori. 
Algebraic curves can be divided into hyperelliptic and nonhyperelliptic curves, and there is a topological obstruction to hyperelliptic minimal surfaces in 3-dimensional flat tori. Actually, a hyperelliptic minimal surface of even genus cannot be minimally immersed into any 3-dimensional flat torus [Meeks 1990, Theorem 3.3].

Every compact Riemann surface can be represented as a branched $d$-cover of the sphere for some $d \geq 1$, and it is reasonable to ask whether there is a topological obstruction or not for $d>2$ (a Riemann surface with $d=2$ is a hyperelliptic curve, and hence we omit the case $d=2$ ). Now we consider this problem for $d=3$. Recall that a nonhyperelliptic curve with $d=3$ is called a trigonal curve.

Our first result is a topological obstruction:

Main Theorem 1.3. Let $f: M_{g} \rightarrow \mathbb{R}^{3} / \Lambda$ be a conformal minimal immersion of a trigonal Riemann surface $M_{g}$ with genus $g$. Then $g=3 r+1$ for some $r \geq 1$. Therefore, a trigonal Riemann surface of genus 0 or $2 \bmod 3$ cannot be minimally immersed into any 3-dimensional flat torus.

Remark 1.4. Among previous examples of trigonal minimal surfaces in 3-dimensional flat tori, we mention the example with $r=1$ from [Shoda 2004] and Schoen's I-WP surface, with $r=3$ [Karcher 1989; Schoen 1970]. Here $r$ is as in Main Theorem 1.3.

Next we consider the higher-codimensional case. Nagano and Smyth [1976] constructed compact minimal surfaces in $n$-dimensional flat tori abstractly, but only few explicit examples are known. We will construct an explicit example of a trigonal minimal surface in the simplest case $(n=4)$, with genus 10 (Section 3). In general, the most difficult part in constructing examples comes from the period condition. It is not always possible to solve the period condition, or even calculate the periods. We overcome this problem through the following process: (i) taking a suitable Riemann surface and considering its symmetries (Construction 3.1), (ii) calculating the periods using functional-theoretic techniques (Lemma 3.4), and (iii) finding a relation between one period and another (Lemma 3.5).

Our example satisfies the following properties:

(a) The conjugate surface $f_{\pi / 2}: M_{10} \rightarrow \mathbb{R}^{4} / \Lambda_{\pi / 2}$ is well defined.

(b) The associate surfaces $f_{\theta}: M_{10} \rightarrow \mathbb{R}^{4} / \Lambda_{\theta}$ are well defined for a countable dense set of angles $e^{i \theta} \subset S^{1}$.

(c) The surface is homologous to 0 in the 4-torus. (Arezzo and Pirola [1999, §6] have shown the existence of minimal surfaces that are not homologous to 0 in the tori.)

Consider these properties in light of the discussion in [Nagano and Smyth 1980]. Let $S_{f}\left(M_{g}\right)$ be a subgroup of the automorphism group of $M_{g}$. We say $f$ has symmetry $S_{f}\left(M_{g}\right)$ if and only if $S_{f}\left(M_{g}\right)$ extends under $f$ to a group of affine 
transformations of $\mathbb{R}^{n} / \Lambda$. When the corresponding linear representation of $S_{f}\left(M_{g}\right)$ is irreducible, we say that $f$ has irreducible symmetry $S_{f}\left(M_{g}\right)$. If the complexification of this representation is also irreducible, we say that $f$ has absolutely irreducible symmetry $S_{f}\left(M_{g}\right)$. Nagano and Smyth [Nagano and Smyth 1980, Theorem 2] showed that if $f$ has absolutely irreducible symmetry, then $f$ satisfies (c); they also showed [Nagano and Smyth 1980, Theorem 5] that if we assume the irreducible conditions above on the part of the Weyl group, together with certain additional conditions, then $f$ satisfies (a) and (b). Our example satisfies (a), (b), (c), but has only reducible symmetry (see Lemma 4.2). Therefore, Nagano and Smyth's irreducibility assumption is sufficient but not necessary.

We summarize our results as follows:

Main Theorem 1.5. There exists a trigonal minimal surface of genus 10 in a 4dimensional flat torus satisfying the following properties:

(i) The conjugate surface $f_{\pi / 2}: M_{10} \rightarrow \mathbb{R}^{4} / \Lambda_{\pi / 2}$ is well defined.

(ii) The associate surfaces $f_{\theta}: M_{10} \rightarrow \mathbb{R}^{4} / \Lambda_{\theta}$ are well defined for a countable dense set of angles $e^{i \theta} \subset S^{1}$.

(iii) The surface is homologous to 0 in the torus and has only reducible symmetry.

\section{A topological obstruction to trigonal minimal surfaces in three-dimensional flat tori}

In this section we prove Main Theorem 1.3. First, we review the spinor representation of minimal surfaces [Kusner and Schmitt 1995]. Let $f: M_{g} \rightarrow \mathbb{R}^{3} / \Lambda$ be a conformal minimal immersion defined by $f(p)=\int_{p_{0}}^{p}\left(\omega_{1}, \omega_{2}, \omega_{3}\right)^{T}$. The Gauss map $G$ makes the diagram

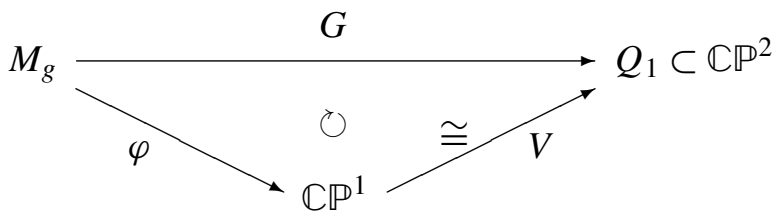

commute, where $V$ is the Veronese embedding, given by

$$
V\left(s_{1}, s_{2}\right)=\left(s_{1}^{2}-s_{2}^{2}, i\left(s_{1}^{2}+s_{2}^{2}\right), 2 s_{1} s_{2}\right)^{T} .
$$

Let $\mathscr{O}_{\mathbb{C P}}(1)$ be the hyperplane bundle on $\mathbb{C P}^{n}$ and $L=\varphi^{*}\left(\mathbb{O}_{\mathbb{C P}}(1)\right)$ its pullback to $M_{g}$. Then $L^{2}=G^{*}\left(\mathbb{O}_{\mathbb{C P}^{2}}(1)\right)$ is the canonical bundle $K$ of $M_{g}$, so $L$ defines a spin structure on $M_{g}$. Again by pullback, we find two holomorphic sections $t_{1}, t_{2}$ of $L$ having no common zeros and such that

$$
\left(\omega_{1}, \omega_{2}, \omega_{3}\right)^{T}=\left(t_{1}^{2}-t_{2}^{2}, i\left(t_{1}^{2}+t_{2}^{2}\right), 2 t_{1} t_{2}\right)^{T} .
$$


The meromorphic function $t_{2} / t_{1}$ can be identified with the usual Gauss map $M_{g} \rightarrow$ $S^{2} \cong \mathbb{C} \cup\{\infty\}$ (see [Hoffman and Osserman 1980]).

Next, we give some notations for a linear series (or system). The standard terminology is as in [Arbarello et al. 1985]. Given a divisor $D$ on $M_{g}$, the complete linear series $|D|$ is the set of effective divisors linearly equivalent to $D$. We have an identification between $|D|$ and projectivization of the space of holomorphic sections of the line bundle defined by $D$ : namely, $|D| \cong P H^{0}\left(M_{g}, O(D)\right)$. Thus a complete linear series is a projective space. More generally, every linear subspace of a complete linear series is called a linear series. A linear series $P W$, where $W$ is a vector subspace of $|D|$, is said to be a $g_{d}^{r}$ if $\operatorname{deg} D=d$ and $\operatorname{dim} W=r+1$. A $g_{d}^{1}$ is called a pencil, a $g_{d}^{2}$ a net, and a $g_{d}^{3}$ a web. We write $t g_{d}^{r}$ for the complete linear series $|t E|$, and $\left|K-g_{d}^{r}\right|$ for the complete linear series $|K-E|$, where $E \in g_{d}^{r}$. By a basepoint of a linear series $P W$ we mean a point common to all divisors of $P W$. If there are none, we say that the linear series is basepoint-free.

Proof of Main Theorem 1.3. We first observe that the degree of the Gauss map is $g-1$ [Meeks 1990, Theorem 3.1]. Hence $M_{g}$ is not trigonal if $g=0,1,2,3$, and we conclude that $g \geq 4$.

We can omit the case $g=4$ because it corresponds to $r=1$. Note that $M_{g}$ is trigonal if and only if there is a basepoint-free pencil $g_{3}^{1}$ on $M_{g}$. If $g>4$, the $g_{3}^{1}$ on $M_{g}$ is a unique complete linear series [Shokurov and Danilov 1994, p. 124].

Let $L$ be the spin bundle given by the spinor representation of $f$ and $D_{L}$ the divisor defined by $L$. Note that Theorem 1.1(1) implies that $\left|D_{L}\right|$ is basepoint-free complete linear series. It is known that every basepoint-free complete linear series $g_{d}^{r}$ is represented by

$$
g_{d}^{r}=r g_{3}^{1} \quad \text { or } \quad\left|K-g_{d}^{r}\right|=r^{\prime} g_{3}^{1}+F \quad\left(r^{\prime}:=g-d+r-1\right),
$$

where $F$ is an effective divisor and consists of the basepoints of $\left|K-g_{d}^{r}\right|$; see [Coppens and Martens 2000, Remark 1.2, Coppens et al. 1992, (1.2.7)]. Applying these facts to $\left|D_{L}\right|=\left|K-D_{L}\right|$, we obtain $\left|D_{L}\right|=r g_{3}^{1}$ for some $r>1$. In particular, $\operatorname{deg} D_{L}=g-1=3 r=\operatorname{deg}\left(r g_{3}^{1}\right)$.

\section{A trigonal minimal surface in a 4-dimensional flat torus}

We now present the example promised in Main Theorem 1.5 and prove its validity, according to the outline given on page 403 .

Construction 3.1. In [Shoda 2004] we constructed a trigonal minimal surface defined by $w^{3}=z^{6}-1$. We now consider the higher-genus version of it. Let $M_{g}$ be the cyclic covering of a line [Miranda 1995, p. 73] given by

$$
w^{3}=z^{g+2}-1 \quad(g=3 r+1, r=1,2,3, \ldots) .
$$


We can find an explicit basis for the space of holomorphic differentials $H^{0}\left(M_{g}, K\right)$ :

$$
H^{0}\left(M_{g}, K\right)=\operatorname{span}\left\{\frac{d z}{w^{2}}, z \frac{d z}{w^{2}}, \ldots, z^{2 r} \frac{d z}{w^{2}}, \frac{d z}{w}, z \frac{d z}{w}, \ldots, z^{r-1} \frac{d z}{w}\right\} .
$$

Note that $M_{g}$ is not well defined if $g=3 r+2$, because Riemann-Hurwitz's formula does not hold, and $M_{g}$ has a cusp singularity at $z=\infty$ if $g=3 r$. We select the simplest case, that is, the case $g=3 r+1$ as above.

Now we consider the following conformal minimal immersion $f$ from $M_{g}$ into a $\mathbb{R}^{4} / \Lambda$ (we will define $\Lambda$ later):

$$
f(p)=\operatorname{Re} \int_{p_{0}}^{p} \underbrace{\left(\frac{1-z^{2 r}}{w^{2}}, \frac{i\left(1+z^{2 r}\right)}{w^{2}}, \frac{z^{2 r-1}+z}{w^{2}}, i \frac{z^{2 r-1}-z}{w^{2}}\right)^{T} d z .}_{\Psi}
$$

Remark 3.2. In [Shoda 2004] we found a component of the moduli space that correspondes to trigonal minimal surfaces in 4-tori. The minimal immersion $f$ defined as above is an element of the component of the moduli space.

To find the symmetries of $f$, we consider the automorphism $\varphi$ defined by

$$
\varphi(z, w)=\left(e^{2 \pi /(3(r+1)) i} z, w\right) .
$$

Then

$$
\varphi^{*} \Psi=e^{2 \pi i / 3}\left(\begin{array}{cc}
R\left(\frac{2 \pi}{3}-\frac{2 \pi}{3(r+1)}\right) & 0 \\
0 & R\left(\frac{4 \pi}{3(r+1)}-\frac{2 \pi}{3}\right)
\end{array}\right) \Psi,
$$

where

$$
R(\theta):=\left(\begin{array}{rr}
\cos \theta & -\sin \theta \\
\sin \theta & \cos \theta
\end{array}\right) .
$$

Remark 3.3. For $r=1,3,7,9$, the value of

$$
\sin \left(\frac{2 \pi}{3}-\frac{2 \pi}{3(r+1)}\right)
$$

is respectively

$$
\frac{\sqrt{3}}{2}, \quad 1, \quad \frac{\sqrt{3}+1}{2 \sqrt{2}}, \quad \sqrt{\frac{5+\sqrt{5}}{8}} .
$$

Since we use $\varphi^{*}$ to calculate periods, we cannot solve the period condition if this sine expression is too complicated. Hence $r=1,3$ may be the best cases for explicit constructions. 
We now consider the case $g=10$, that is, $r=3$. Then $M_{10}$ is defined by $w^{3}=z^{12}-1$ and $f$ reduces to

$$
f(p)=\operatorname{Re} \int_{p_{0}}^{p} \underbrace{\left(\frac{1-z^{6}}{w^{2}}, \frac{i\left(1+z^{6}\right)}{w^{2}}, \frac{z^{5}+z}{w^{2}}, \frac{i\left(z^{5}-z\right)}{w^{2}}\right)^{T} d z .}_{\Psi}
$$

The map $\varphi$ is given by $\varphi(z, w)=\left(e^{\pi i / 6} z, w\right)$ and

$$
\varphi^{*} \Psi=e^{2 \pi i / 3}\left(\begin{array}{cc}
R\left(\frac{\pi}{2}\right) & 0 \\
0 & R\left(-\frac{\pi}{3}\right)
\end{array}\right) \Psi=e^{2 \pi i / 3}\left(\begin{array}{cccc}
0 & -1 & 0 & 0 \\
1 & 0 & 0 & 0 \\
0 & 0 & \frac{1}{2} & \frac{\sqrt{3}}{2} \\
0 & 0 & -\frac{\sqrt{3}}{2} & \frac{1}{2}
\end{array}\right) \Psi .
$$

We next prove the well-definedness of the following conformal minimal immersion of $M_{10}$ into $\mathbb{R}^{4} / \Lambda$ :

$$
\begin{aligned}
f: M_{10} & \rightarrow \mathbb{R}^{4} / \Lambda \\
& p \longmapsto \operatorname{Re} \int_{p_{0}}^{p}\left(\frac{1-z^{6}}{w^{2}}, \frac{i\left(1+z^{6}\right)}{w^{2}}, \frac{z^{5}+z}{w^{2}}, \frac{i\left(z^{5}-z\right)}{w^{2}}\right)^{T} d z \\
& \left(w^{3}=z^{12}-1\right),
\end{aligned}
$$

where $\Lambda$ is given by the beta function $B(a, b)$ :

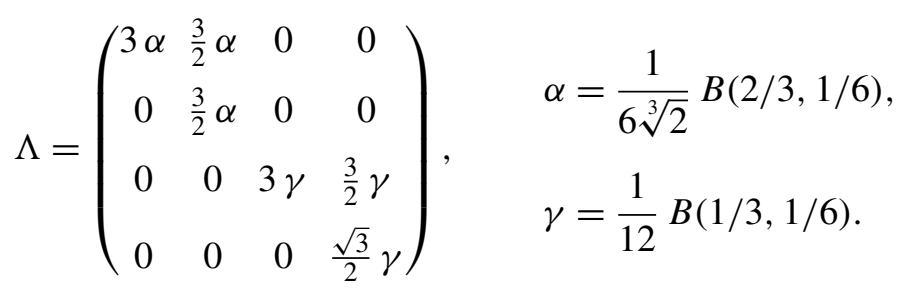

Periods. We now calculate the periods of $f$ defined by (4), and consider the conjugate surface and associate surfaces. The 1 -cycles $A_{1}, A_{2}, \ldots, A_{10}, B_{1}, B_{2}, \ldots$, $B_{10}$ are established by defining

$$
\begin{gathered}
A_{1}=\left\{(z, w)=\left(e^{i t}, w(t)\right) \mid t \in\left[0, \frac{\pi}{6}\right], w\left(\frac{\pi}{12}\right)=-\sqrt[3]{2}\right\} \\
\cup\left\{(z, w)=\left(e^{-i t}, w(t)\right) \mid t \in\left[-\frac{\pi}{6}, 0\right], w\left(-\frac{\pi}{12}\right)=-\sqrt[3]{2} e^{2 \pi i / 3}\right\}, \\
A_{2}=\left\{(z, w)=\left(e^{i t}, w(t)\right) \mid t \in\left[0, \frac{\pi}{6}\right], w\left(\frac{\pi}{12}\right)=-\sqrt[3]{2}\right\} \\
\cup\left\{(z, w)=\left(e^{-i t}, w(t)\right) \mid t \in\left[-\frac{\pi}{6}, 0\right], w\left(-\frac{\pi}{12}\right)=-\sqrt[3]{2} e^{4 \pi i / 3}\right\},
\end{gathered}
$$


and then taking successive images under $\varphi$ :

$$
A_{k+2}=\varphi^{2}\left(A_{k}\right) \quad(1 \leq k \leq 8), \quad B_{k}=\varphi\left(A_{k}\right) \quad(1 \leq k \leq 10) .
$$

Lemma 3.4. The periods along $A_{1}$ and $A_{2}$ are given by

$$
\begin{aligned}
& \left(\begin{array}{c}
\int_{A_{1}} \frac{1-z^{6}}{w^{2}} d z \\
\int_{A_{1}} \frac{i\left(1+z^{6}\right)}{w^{2}} d z \\
\int_{A_{1}} \frac{z^{5}+z}{w^{2}} d z \\
\int_{A_{1}} \frac{i\left(z^{5}-z\right)}{w^{2}} d z
\end{array}\right)=\left(\begin{array}{c}
\frac{1+e^{\pi i / 3}}{6 \sqrt[3]{2}} B(2 / 3,1 / 6) \\
-\frac{1+e^{\pi i / 3}}{6 \sqrt[3]{2}} B(2 / 3,1 / 6) \\
\frac{i\left(1+e^{\pi i / 3}\right)}{4 \sqrt{3}} B(1 / 3,1 / 6) \\
-i\left(1+e^{\pi i / 3}\right) \int_{1 / 2}^{1} \frac{d t}{\sqrt[3]{4\left(1-t^{2}\right)\left(4 t^{2}-1\right)^{2}}}
\end{array}\right), \\
& \left(\begin{array}{c}
\int_{A_{2}} \frac{1-z^{6}}{w^{2}} d z \\
\int_{A_{2}} \frac{i\left(1+z^{6}\right)}{w^{2}} d z \\
\int_{A_{2}} \frac{z^{5}+z}{w^{2}} d z \\
\int_{A_{2}} \frac{i\left(z^{5}-z\right)}{w^{2}} d z
\end{array}\right)=\left(\begin{array}{c}
\frac{e^{2 \pi i / 3}+e^{\pi i / 3}}{6 \sqrt[3]{2}} B(2 / 3,1 / 6) \\
-\frac{e^{2 \pi i / 3}+e^{\pi i / 3}}{6 \sqrt[3]{2}} B(2 / 3,1 / 6) \\
\frac{i\left(e^{\pi i / 3}+e^{2 \pi i / 3}\right)}{4 \sqrt{3}} B(1 / 3,1 / 6) \\
-i\left(e^{\pi i / 3}+e^{2 \pi i / 3}\right) \int_{1 / 2}^{1} \frac{d t}{\sqrt[3]{4\left(1-t^{2}\right)\left(4 t^{2}-1\right)^{2}}}
\end{array}\right)
\end{aligned}
$$

Proof. A1-period, integrand $\left(1-z^{6}\right) / w^{2}$. For $t \in[0, \pi / 6]$ we set

$$
\eta=\frac{1}{2}\left(z^{3}+\frac{1}{z^{3}}\right)=\frac{1}{2}\left(e^{3 i t}+e^{-3 i t}\right)=\cos 3 t .
$$

Then $d \eta=3 \frac{z^{6}-1}{2 z^{4}} d z$, so

$$
\frac{1-z^{6}}{w^{2}} d z=\frac{1-z^{6}}{w^{2}} \frac{2 z^{4}}{3\left(z^{6}-1\right)} d \eta=-\frac{2}{3} \frac{z^{4}}{w^{2}} d \eta .
$$

To calculate $z^{4} / w^{2}$, we consider

$$
\left(\frac{z^{4}}{w^{2}}\right)^{3}=\frac{z^{12}}{\left(z^{12}-1\right)^{2}}=\frac{1}{z^{12}+z^{-12}-2} .
$$

Note that $z^{6}+\frac{1}{z^{6}}=4 \eta^{2}-2$ and thus

$$
z^{12}+\frac{1}{z^{12}}=\left(4 \eta^{2}-2\right)^{2}-2=16 \eta^{4}-16 \eta^{2}+2 .
$$


Hence

$$
\left(\frac{z^{4}}{w^{2}}\right)^{3}=-\frac{1}{16 \eta^{2}\left(1-\eta^{2}\right)}<0,
$$

and we can take a suitable branch for $z^{4} / w^{2}$ :

$$
\frac{z^{4}}{w^{2}}\left(\frac{\pi}{12}\right)=\frac{e^{\pi i / 3}}{(-\sqrt[3]{2})^{2}}=\frac{e^{\pi i / 3}}{\sqrt[3]{4}}
$$

Choosing the branch $\sqrt[3]{\eta^{2}\left(1-\eta^{2}\right)}>0$, we get

$$
\frac{z^{4}}{w^{2}}=\frac{e^{\pi i / 3}}{2 \sqrt[3]{2} \sqrt[3]{\eta^{2}\left(1-\eta^{2}\right)}}
$$

It follows that

$$
\frac{1-z^{6}}{w^{2}} d z=-\frac{1}{3 \sqrt[3]{2}} \frac{e^{\pi i / 3}}{\sqrt[3]{\eta^{2}\left(1-\eta^{2}\right)}} d \eta
$$

For $t \in[-\pi / 6,0]$ we obtain, through similar arguments,

$$
\frac{1-z^{6}}{w^{2}} d z=\frac{1}{3 \sqrt[3]{2}} \frac{1}{\sqrt[3]{\eta^{2}\left(1-\eta^{2}\right)}} d \eta
$$

Hence

$$
\begin{aligned}
\int_{A_{1}} \frac{1-z^{6}}{w^{2}} d z & =\int_{1}^{0}-\frac{1}{3 \sqrt[3]{2}} \frac{e^{\pi i / 3}}{\sqrt[3]{\eta^{2}\left(1-\eta^{2}\right)}} d \eta+\int_{0}^{1} \frac{1}{3 \sqrt[3]{2}} \frac{1}{\sqrt[3]{\eta^{2}\left(1-\eta^{2}\right)}} d \eta \\
& =\frac{1+e^{\pi i / 3}}{3 \sqrt[3]{2}} \int_{0}^{1} \frac{d \eta}{\sqrt[3]{\eta^{2}\left(1-\eta^{2}\right)}} \underbrace{=}_{t=\eta^{2}} \frac{1+e^{\pi i / 3}}{6 \sqrt[3]{2}} \int_{0}^{1} t^{-5 / 6}(1-t)^{-1 / 3} d t \\
& =\frac{1+e^{\pi i / 3}}{6 \sqrt[3]{2}} B(2 / 3,1 / 6)
\end{aligned}
$$

$\underline{A_{2}-\text { period, integrand }\left(1-z^{6}\right) / w^{2}}$. This is calculated similarly:

$$
\begin{aligned}
\int_{A_{2}} \frac{1-z^{6}}{w^{2}} d z & =\int_{1}^{0}-\frac{1}{3 \sqrt[3]{2}} \frac{e^{\pi i / 3}}{\sqrt[3]{\eta^{2}\left(1-\eta^{2}\right)}} d \eta+\int_{0}^{1} \frac{1}{3 \sqrt[3]{2}} \frac{e^{2 \pi i / 3}}{\sqrt[3]{\eta^{2}\left(1-\eta^{2}\right)}} d \eta \\
& =\frac{e^{2 \pi i / 3}+e^{\pi i / 3}}{3 \sqrt[3]{2}} \int_{0}^{1} \frac{d \eta}{\sqrt[3]{\eta^{2}\left(1-\eta^{2}\right)}}=\frac{e^{2 \pi i / 3}+e^{\pi i / 3}}{6 \sqrt[3]{2}} B(2 / 3,1 / 6) .
\end{aligned}
$$

$\underline{A_{1} \text {-period, integrand } i\left(1+z^{6}\right) / w^{2}}$. For $t \in[0, \pi / 6]$ we set

$$
\eta=-\frac{i}{2}\left(z^{3}-\frac{1}{z^{3}}\right)=-\frac{i}{2}\left(e^{3 i t}-e^{-3 i t}\right)=\sin 3 t
$$


Then $d \eta=-3 i \frac{z^{6}+1}{2 z^{4}} d z$, so

$$
\frac{i\left(1+z^{6}\right)}{w^{2}} d z=\frac{i\left(1+z^{6}\right)}{w^{2}} \frac{2 z^{4}}{-3 i\left(z^{6}+1\right)} d \eta=-\frac{2}{3} \frac{z^{4}}{w^{2}} d \eta .
$$

To calculate $z^{4} / w^{2}$, we consider

$$
\left(\frac{z^{4}}{w^{2}}\right)^{3}=\frac{z^{12}}{\left(z^{12}-1\right)^{2}}=\frac{1}{z^{12}+z^{-12}-2} .
$$

Note that $z^{6}+\frac{1}{z^{6}}=-4 \eta^{2}+2$ and thus

$$
z^{12}+\frac{1}{z^{12}}=\left(-4 \eta^{2}+2\right)^{2}-2=16 \eta^{4}-16 \eta^{2}+2 .
$$

Hence

$$
\left(\frac{z^{4}}{w^{2}}\right)^{3}=-\frac{1}{16 \eta^{2}\left(1-\eta^{2}\right)}<0,
$$

and we take the branch

$$
\frac{z^{4}}{w^{2}}\left(\frac{\pi}{12}\right)=\frac{e^{\pi i / 3}}{(-\sqrt[3]{2})^{2}}=\frac{e^{\pi i / 3}}{\sqrt[3]{4}} .
$$

Choosing the branch $\sqrt[3]{\eta^{2}\left(1-\eta^{2}\right)}>0$, we get

$$
\frac{z^{4}}{w^{2}}=\frac{e^{\pi i / 3}}{2 \sqrt[3]{2} \sqrt[3]{\eta^{2}\left(1-\eta^{2}\right)}} .
$$

It follows that

$$
\frac{i\left(1+z^{6}\right)}{w^{2}} d z=-\frac{1}{3 \sqrt[3]{2}} \frac{e^{\pi i / 3}}{\sqrt[3]{\eta^{2}\left(1-\eta^{2}\right)}} d \eta .
$$

For $t \in[-\pi / 6,0]$ we obtain

$$
\frac{i\left(1+z^{6}\right)}{w^{2}} d z=\frac{1}{3 \sqrt[3]{2}} \frac{1}{\sqrt[3]{\eta^{2}\left(1-\eta^{2}\right)}} d \eta
$$

so

$$
\begin{aligned}
\int_{A_{1}} \frac{i\left(1+z^{6}\right)}{w^{2}} d z & =\int_{0}^{1}-\frac{1}{3 \sqrt[3]{2}} \frac{e^{\pi i / 3}}{\sqrt[3]{\eta^{2}\left(1-\eta^{2}\right)}} d \eta+\int_{1}^{0} \frac{1}{3 \sqrt[3]{2}} \frac{1}{\sqrt[3]{\eta^{2}\left(1-\eta^{2}\right)}} d \eta \\
& =-\frac{1+e^{\pi i / 3}}{3 \sqrt[3]{2}} \int_{0}^{1} \frac{d \eta}{\sqrt[3]{\eta^{2}\left(1-\eta^{2}\right)}}=-\frac{1+e^{\pi i / 3}}{6 \sqrt[3]{2}} B(2 / 3,1 / 6)
\end{aligned}
$$


$A_{2}$-period, integrand $i\left(1+z^{6}\right) / w^{2}$.

$$
\begin{aligned}
\int_{A_{2}} \frac{i\left(1+z^{6}\right)}{w^{2}} & d z=\int_{0}^{1}-\frac{1}{3 \sqrt[3]{2}} \frac{e^{\pi i / 3}}{\sqrt[3]{\eta^{2}\left(1-\eta^{2}\right)}} d \eta+\int_{1}^{0} \frac{1}{3 \sqrt[3]{2}} \frac{e^{2 \pi i / 3}}{\sqrt[3]{\eta^{2}\left(1-\eta^{2}\right)}} d \eta \\
= & -\frac{e^{2 \pi i / 3}+e^{\pi i / 3}}{3 \sqrt[3]{2}} \int_{0}^{1} \frac{d \eta}{\sqrt[3]{\eta^{2}\left(1-\eta^{2}\right)}}=-\frac{e^{2 \pi i / 3}+e^{\pi i / 3}}{6 \sqrt[3]{2}} B(2 / 3,1 / 6) .
\end{aligned}
$$

$\underline{A_{1} \text {-period, integrand }\left(z^{5}+z\right) / w^{2}}$. For $t \in[0, \pi / 6]$ we set

$$
\eta=\frac{1}{2 i}\left(z^{2}-\frac{1}{z^{2}}\right)=\sin 2 t .
$$

Then $d \eta=\frac{1+z^{4}}{i z^{3}} d z$, so

$$
\frac{z^{5}+z}{w^{2}} d z=\frac{z\left(1+z^{4}\right)}{w^{2}} \frac{i z^{3}}{1+z^{4}} d \eta=i \frac{z^{4}}{w^{2}} d \eta .
$$

To calculate $z^{4} / w^{2}$, we consider

$$
\left(\frac{z^{4}}{w^{2}}\right)^{3}=\frac{z^{12}}{\left(z^{12}-1\right)^{2}}=\frac{1}{z^{12}+z^{-12}-2} .
$$

Note that $z^{2}-z^{-2}=2 i \eta$ and $z^{4}+z^{-4}=2-4 \eta^{2}$, hence $z^{8}+z^{-8}=\left(2-4 \eta^{2}\right)^{2}-2=$ $2-16 \eta^{2}+16 \eta^{4}$, and finally

$$
z^{12}+\frac{1}{z^{12}}-2=-4 \eta^{2}\left(3-4 \eta^{2}\right)^{2}
$$

Therefore

$$
\left(\frac{z^{4}}{w^{2}}\right)^{3}=-\frac{1}{4 \eta^{2}\left(3-4 \eta^{2}\right)^{2}}<0,
$$

and we take the branch

$$
\frac{z^{4}}{w^{2}}\left(\frac{\pi}{12}\right)=\frac{e^{\pi i / 3}}{\sqrt[3]{4}}
$$

Choosing the branch $\sqrt[3]{4 \eta^{2}\left(3-4 \eta^{2}\right)^{2}}>0$, we get

$$
\frac{z^{4}}{w^{2}}=\frac{e^{\pi i / 3}}{\sqrt[3]{4 \eta^{2}\left(3-4 \eta^{2}\right)^{2}}} .
$$

It follows that

$$
\frac{z^{5}+z}{w^{2}} d z=i \frac{e^{\pi i / 3}}{\sqrt[3]{4 \eta^{2}\left(3-4 \eta^{2}\right)^{2}}} d \eta
$$

For $t \in[-\pi / 6,0]$ we obtain

$$
\frac{z^{5}+z}{w^{2}} d z=i \frac{-1}{\sqrt[3]{4 \eta^{2}\left(3-4 \eta^{2}\right)^{2}}} d \eta
$$


So

$$
\begin{aligned}
\int_{A_{1}} \frac{z^{5}+z}{w^{2}} d z & =\int_{0}^{\sqrt{3} / 2} i \frac{e^{\pi i / 3}}{\sqrt[3]{4 \eta^{2}\left(3-4 \eta^{2}\right)^{2}}} d \eta+\int_{\sqrt{3} / 2}^{0} i \frac{-1}{\sqrt[3]{4 \eta^{2}\left(3-4 \eta^{2}\right)^{2}}} d \eta \\
& =i\left(1+e^{\pi i / 3}\right) \int_{0}^{\sqrt{3} / 2} \frac{d \eta}{\sqrt[3]{4 \eta^{2}\left(3-4 \eta^{2}\right)^{2}}} \\
& =\frac{i\left(1+e^{\pi i / 3}\right)}{2 \sqrt{3}} \int_{0}^{1} \frac{d t}{\sqrt[3]{t^{2}\left(1-t^{2}\right)^{2}}} \quad\left(\eta=\frac{\sqrt{3}}{2} t\right) \\
& =\frac{i\left(1+e^{\pi i / 3}\right)}{4 \sqrt{3}} \int_{0}^{1} s^{-5 / 6}(1-s)^{-2 / 3} d s \quad\left(s=t^{2}\right) \\
& =\frac{i\left(1+e^{\pi i / 3}\right)}{4 \sqrt{3}} B(1 / 3,1 / 6) .
\end{aligned}
$$

$\underline{A_{2}-\text { period, integrand }\left(z^{5}+z\right) / w^{2}}$.

$$
\begin{aligned}
& \int_{A_{2}} \frac{z^{5}+z}{w^{2}} d z=\int_{0}^{\sqrt{3} / 2} i \frac{e^{\pi i / 3}}{\sqrt[3]{4 \eta^{2}\left(3-4 \eta^{2}\right)^{2}}} d \eta+\int_{\sqrt{3} / 2}^{0} i \frac{-e^{2 \pi i / 3}}{\sqrt[3]{4 \eta^{2}\left(3-4 \eta^{2}\right)^{2}}} d \eta \\
& =i\left(e^{\pi i / 3}+e^{2 \pi i / 3}\right) \int_{0}^{\sqrt{3} / 2} \frac{d \eta}{\sqrt[3]{4 \eta^{2}\left(3-4 \eta^{2}\right)^{2}}}=\frac{i\left(e^{\pi i / 3}+e^{2 \pi i / 3}\right)}{4 \sqrt{3}} B(1 / 3,1 / 6) .
\end{aligned}
$$

$\underline{A_{1} \text {-period, integrand } i\left(z^{5}-z\right) / w^{2}}$. For $t \in[0, \pi / 6]$ we set

$$
\eta=\frac{1}{2}\left(z^{2}+\frac{1}{z^{2}}\right)=\cos 2 t
$$

Then $d \eta=-\frac{1-z^{4}}{z^{3}} d z$, so

$$
\frac{i\left(z^{5}-z\right)}{w^{2}} d z=\frac{-i z\left(1-z^{4}\right)}{w^{2}} \frac{-z^{3}}{1-z^{4}} d \eta=i \frac{z^{4}}{w^{2}} d \eta
$$

To calculate $z^{4} / w^{2}$, we consider

$$
\left(\frac{z^{4}}{w^{2}}\right)^{3}=\frac{z^{12}}{\left(z^{12}-1\right)^{2}}=\frac{1}{z^{12}+z^{-12}-2}
$$

Now $z^{2}+z^{-2}=2 \eta, z^{4}+z^{-4}=4 \eta^{2}-2$, hence $z^{6}+z^{-6}=\left(z^{2}+z^{-2}\right)\left(z^{4}-1+z^{-4}\right)=$ $2 \eta\left(4 \eta^{2}-3\right)$, and finally

$$
z^{12}+\frac{1}{z^{12}}-2=\left(2 \eta\left(4 \eta^{2}-3\right)\right)^{2}-4=-4\left(1-\eta^{2}\right)\left(4 \eta^{2}-1\right)^{2}
$$

Therefore

$$
\left(\frac{z^{4}}{w^{2}}\right)^{3}=-\frac{1}{4\left(1-\eta^{2}\right)\left(4 \eta^{2}-1\right)^{2}}<0
$$


and we take the branch

$$
\frac{z^{4}}{w^{2}}\left(\frac{\pi}{12}\right)=\frac{e^{\pi i / 3}}{\sqrt[3]{4}}
$$

Choosing the branch $\sqrt[3]{4\left(1-\eta^{2}\right)\left(4 \eta^{2}-1\right)^{2}}>0$, we get

$$
\frac{z^{4}}{w^{2}}=\frac{e^{\pi i / 3}}{\sqrt[3]{4\left(1-\eta^{2}\right)\left(4 \eta^{2}-1\right)^{2}}} .
$$

It follows that

$$
\frac{i\left(z^{5}-z\right)}{w^{2}} d z=i \frac{e^{\pi i / 3}}{\sqrt[3]{4\left(1-\eta^{2}\right)\left(4 \eta^{2}-1\right)^{2}}} d \eta .
$$

For $t \in[-\pi / 6,0]$ we obtain

so

$$
\frac{i\left(z^{5}-z\right)}{w^{2}} d z=i \frac{-1}{\sqrt[3]{4\left(1-\eta^{2}\right)\left(4 \eta^{2}-1\right)^{2}}} d \eta
$$

$$
\begin{aligned}
\int_{A_{1}} \frac{i\left(z^{5}-z\right)}{w^{2}} & d z \\
= & \int_{1}^{1 / 2} i \frac{e^{\pi i / 3}}{\sqrt[3]{4\left(1-\eta^{2}\right)\left(4 \eta^{2}-1\right)^{2}}} d \eta+\int_{1 / 2}^{1} i \frac{-1}{\sqrt[3]{4\left(1-\eta^{2}\right)\left(4 \eta^{2}-1\right)^{2}}} d \eta \\
= & -i\left(1+e^{\pi i / 3}\right) \int_{1 / 2}^{1} \frac{d t}{\sqrt[3]{4\left(1-t^{2}\right)\left(4 t^{2}-1\right)^{2}}}
\end{aligned}
$$

$A_{2}$-period, integrand $i\left(z^{5}-z\right) / w^{2}$.

$$
\begin{aligned}
\int_{A_{2}} \frac{i\left(z^{5}-z\right)}{w^{2}} & d z \\
= & \int_{1}^{1 / 2} i \frac{e^{\pi i / 3}}{\sqrt[3]{4\left(1-\eta^{2}\right)\left(4 \eta^{2}-1\right)^{2}}} d \eta+\int_{1 / 2}^{1} i \frac{-e^{2 \pi i / 3}}{\sqrt[3]{4\left(1-\eta^{2}\right)\left(4 \eta^{2}-1\right)^{2}}} d \eta \\
= & -i\left(e^{\pi i / 3}+e^{2 \pi i / 3}\right) \int_{1 / 2}^{1} \frac{d t}{\sqrt[3]{4\left(1-t^{2}\right)\left(4 t^{2}-1\right)^{2}}}
\end{aligned}
$$

Lemma 3.5. Set

$\alpha=\frac{1}{6 \sqrt[3]{2}} B(2 / 3,1 / 6), \quad \beta=\frac{1}{4 \sqrt{3}} B(1 / 3,1 / 6), \quad \gamma=\int_{1 / 2}^{1} \frac{d t}{\sqrt[3]{4\left(1-t^{2}\right)\left(4 t^{2}-1\right)^{2}}}$.

Then

$$
\beta=\sqrt{3} \gamma
$$

Proof. We consider the period of $\left(z^{5}+z\right) / w^{2} d z$ along $B_{5}=\varphi^{5}\left(A_{1}\right)$. The arguments below are similar to those used to prove Lemma 3.4. For $t \in[5 \pi / 6, \pi]$ we set $\eta=\sin 2 t$. Now 


$$
\frac{z^{4}}{w^{2}}\left(\frac{11}{12} \pi\right)=\frac{e^{11 \pi i / 3}}{\sqrt[3]{4}}=-\frac{e^{2 \pi i / 3}}{\sqrt[3]{4}},
$$

so choosing a branch $\sqrt[3]{4 \eta^{2}\left(3-4 \eta^{2}\right)^{2}}>0$, we get

$$
\frac{z^{5}+z}{w^{2}} d z=i \frac{-e^{2 \pi i / 3}}{\sqrt[3]{4 \eta^{2}\left(3-4 \eta^{2}\right)^{2}}} d \eta
$$

Similarly, for $t \in[-\pi,-5 \pi / 6]$, we obtain

It follows that

$$
\frac{z^{5}+z}{w^{2}} d z=i \frac{e^{\pi i / 3}}{\sqrt[3]{4 \eta^{2}\left(3-4 \eta^{2}\right)^{2}}} d \eta
$$

(5)

$$
\begin{aligned}
\int_{B_{5}} \frac{z^{5}+z}{w^{2}} d z & =\int_{-\sqrt{3} / 2}^{0} i \frac{-e^{2 \pi i / 3}}{\sqrt[3]{4 \eta^{2}\left(3-4 \eta^{2}\right)^{2}}} d \eta+\int_{0}^{-\sqrt{3} / 2} i \frac{e^{\pi i / 3}}{\sqrt[3]{4 \eta^{2}\left(3-4 \eta^{2}\right)^{2}}} d \eta \\
& =-i\left(e^{2 \pi i / 3}+e^{\pi i / 3}\right) \beta
\end{aligned}
$$

On the other hand, using the action of $\varphi$, we obtain

$$
\left(\varphi^{*}\right)^{5}=e^{10 \pi i / 3}\left(\begin{array}{cc}
R\left(\frac{\pi}{2}\right) & 0 \\
0 & R\left(-\frac{\pi}{3}\right)
\end{array}\right)^{5}=-e^{\pi i / 3}\left(\begin{array}{cccc}
0 & -1 & 0 & 0 \\
1 & 0 & 0 & 0 \\
0 & 0 & \frac{1}{2} & -\frac{\sqrt{3}}{2} \\
0 & 0 & \frac{\sqrt{3}}{2} & \frac{1}{2}
\end{array}\right) .
$$

Hence,

$$
\begin{aligned}
\int_{B_{5}} \frac{z^{5}+z}{w^{2}} d z & =\int_{\varphi^{5}\left(A_{1}\right)} \frac{z^{5}+z}{w^{2}} d z=\int_{A_{1}}\left(\varphi^{*}\right)^{5}\left(\frac{z^{5}+z}{w^{2}} d z\right) \\
& =-e^{\pi i / 3}\left(\frac{1}{2}-\frac{\sqrt{3}}{2}\right)\left(\begin{array}{c}
\int_{A_{1}} \frac{z^{5}+z}{w^{2}} d z \\
\int_{A_{1}} \frac{i\left(z^{5}-z\right)}{w^{2}} d z
\end{array}\right) \\
& =-e^{\pi i / 3}\left(\frac{1}{2}-\frac{\sqrt{3}}{2}\right)\left(\begin{array}{c}
i\left(1+e^{\pi i / 3}\right) \beta \\
-i\left(1+e^{\pi i / 3}\right) \gamma
\end{array}\right) \\
& =-e^{\pi i / 3}\left(\frac{i}{2}\left(1+e^{\pi i / 3}\right) \beta+\frac{\sqrt{3}}{2} i\left(1+e^{\pi i / 3}\right) \gamma\right) \\
& =-i\left(e^{\pi i / 3}+e^{2 \pi i / 3}\right)\left(\frac{\beta}{2}+\frac{\sqrt{3}}{2} \gamma\right) .
\end{aligned}
$$


The equality of (5) and (6) implies the claim of the lemma.

Using the equality $\beta=\sqrt{3} \gamma$, Lemma 3.4, and the action of $\varphi$, we can write the period matrix $\operatorname{Re} \Omega$ of $f$ in terms of the quantities $\alpha, \gamma$ introduced in Lemma 3.5:

$$
\operatorname{Re} \Omega=\operatorname{Re}\left(\Omega_{1}, \Omega_{2}, \Omega_{3}, \Omega_{4}, \Omega_{5}, \Omega_{6}, \Omega_{7}\right),
$$

where

$$
\begin{aligned}
& \Omega_{1}=\left(\left(1+e^{\pi i / 3}\right)\left(\begin{array}{c}
\alpha \\
-\alpha \\
\sqrt{3} i \gamma \\
-i \gamma
\end{array}\right),\left(e^{2 \pi i / 3}+e^{\pi i / 3}\right)\left(\begin{array}{c}
\alpha \\
-\alpha \\
\sqrt{3} i \gamma \\
-i \gamma
\end{array}\right),\left(-1+e^{2 \pi i / 3}\right)\left(\begin{array}{c}
\alpha \\
\alpha \\
0 \\
-2 i \gamma
\end{array}\right)\right), \\
& \Omega_{2}=\left(-\left(1+e^{\pi i / 3}\right)\left(\begin{array}{c}
\alpha \\
\alpha \\
0 \\
-2 i \gamma
\end{array}\right),\left(e^{2 \pi i / 3}+e^{\pi i / 3}\right)\left(\begin{array}{c}
\alpha \\
-\alpha \\
\sqrt{3} i \gamma \\
i \gamma
\end{array}\right),\left(-1+e^{2 \pi i / 3}\right)\left(\begin{array}{c}
\alpha \\
-\alpha \\
\sqrt{3} i \gamma \\
i \gamma
\end{array}\right)\right) \text {, } \\
& \Omega_{3}=\left(-\left(1+e^{\pi i / 3}\right)\left(\begin{array}{c}
\alpha \\
\alpha \\
\sqrt{3} i \gamma \\
-i \gamma
\end{array}\right),-\left(e^{2 \pi i / 3}+e^{\pi i / 3}\right)\left(\begin{array}{c}
\alpha \\
\alpha \\
\sqrt{3} i \gamma \\
-i \gamma
\end{array}\right),\left(-1+e^{2 \pi i / 3}\right)\left(\begin{array}{c}
\alpha \\
-\alpha \\
0 \\
2 i \gamma
\end{array}\right)\right) \text {, } \\
& \Omega_{4}=\left(-\left(1+e^{\pi i / 3}\right)\left(\begin{array}{c}
\alpha \\
-\alpha \\
0 \\
2 i \gamma
\end{array}\right),-\left(e^{2 \pi i / 3}+e^{\pi i / 3}\right)\left(\begin{array}{c}
\alpha \\
\alpha \\
\sqrt{3} i \gamma \\
i \gamma
\end{array}\right),\left(1-e^{2 \pi i / 3}\right)\left(\begin{array}{c}
\alpha \\
\alpha \\
\sqrt{3} i \gamma \\
i \gamma
\end{array}\right)\right) \text {, } \\
& \Omega_{5}=\left(-\left(1+e^{\pi i / 3}\right)\left(\begin{array}{c}
\alpha \\
-\alpha \\
-\sqrt{3} i \gamma \\
i \gamma
\end{array}\right),-\left(e^{2 \pi i / 3}+e^{\pi i / 3}\right)\left(\begin{array}{c}
\alpha \\
-\alpha \\
-\sqrt{3} i \gamma \\
i \gamma
\end{array}\right),\left(1-e^{2 \pi i / 3}\right)\left(\begin{array}{c}
\alpha \\
\alpha \\
0 \\
2 i \gamma
\end{array}\right)\right) \text {, } \\
& \Omega_{6}=\left(\left(1+e^{\pi i / 3}\right)\left(\begin{array}{c}
\alpha \\
\alpha \\
0 \\
2 i \gamma
\end{array}\right),-\left(e^{2 \pi i / 3}+e^{\pi i / 3}\right)\left(\begin{array}{c}
\alpha \\
-\alpha \\
-\sqrt{3} i \gamma \\
-i \gamma
\end{array}\right),\left(1-e^{2 \pi i / 3}\right)\left(\begin{array}{c}
\alpha \\
-\alpha \\
-\sqrt{3} i \gamma \\
-i \gamma
\end{array}\right)\right) \text {, } \\
& \left.\Omega_{7}=\left(1+e^{\pi i / 3}\right)\left(\begin{array}{c}
\alpha \\
\alpha \\
-\sqrt{3} i \gamma \\
i \gamma
\end{array}\right),\left(e^{2 \pi i / 3}+e^{\pi i / 3}\right)\left(\begin{array}{c}
\alpha \\
\alpha \\
-\sqrt{3} i \gamma \\
i \gamma
\end{array}\right)\right) .
\end{aligned}
$$


The lattices $\boldsymbol{\Lambda}$ and $\boldsymbol{\Lambda}_{\pi / 2}$. To complete the construction of our minimal surface, we still need to show that the periods form a lattice; in the same step we will also show the well-definedness of the conjugate, claimed by the Main Theorem 1.5(i). Recall that a lattice in a real vector space $\mathbb{R}^{n}$ is a discrete subgroup of maximal rank in $\mathbb{R}^{n}$; a set of lattice vectors is any set that generates the lattice as an abelian group. Given a spanning set $\left\{u_{1}, u_{2}, \ldots, u_{m}\right\}$ of $\mathbb{R}^{n}$ (so $m \geq n$ ), it is easy to see that $\left\{u_{1}, u_{2}, \ldots, u_{m}\right\}$ is a set of lattice vectors if and only if there exists a set $\left\{v_{1}, v_{2}, \ldots, v_{n}\right\}$ of lattice vectors and integer matrices $G_{1}$ and $G_{2}$ (respectively $m \times n$ and $n \times m$ in shape) such that

$$
\begin{aligned}
& \left\{v_{1}, v_{2}, \ldots, v_{n}\right\}=\left\{u_{1}, u_{2}, \ldots, u_{m}\right\} G_{1}, \\
& \left\{u_{1}, u_{2}, \ldots, u_{m}\right\}=\left\{v_{1}, v_{2}, \ldots, v_{n}\right\} G_{2} .
\end{aligned}
$$

To determine the lattice $\Lambda$ of our example, we first define the four matrices

$$
\begin{aligned}
& \Omega_{8}=\left(\begin{array}{cccc}
-2\left(e^{2 \pi i / 3}-1\right) \alpha & 2\left(1+e^{\pi i / 3}\right) \alpha & \left(e^{\pi i / 3}+e^{2 \pi i / 3}\right) \alpha & -\left(1+e^{\pi i / 3}\right) \alpha \\
0 & 0 & -\left(e^{\pi i / 3}+e^{2 \pi i / 3}\right) \alpha & \left(1+e^{\pi i / 3}\right) \alpha \\
0 & 0 & 0 & 0 \\
0 & 0 & 0 & 0
\end{array}\right), \\
& \Omega_{9}=\left(\begin{array}{cccc}
0 & 0 & 0 & 0 \\
0 & 0 & 0 & 0 \\
-2 \sqrt{3} i\left(e^{\pi i / 3}+e^{2 \pi i / 3}\right) \gamma & 0 & \sqrt{3} i\left(1+e^{\pi i / 3}\right) \gamma & \sqrt{3} i\left(e^{\pi i / 3}+e^{2 \pi i / 3}\right) \gamma \\
0 & 2 i\left(1+e^{\pi i / 3}\right) \gamma & i\left(1+e^{\pi i / 3}\right) \gamma & -i\left(e^{\pi i / 3}+e^{2 \pi i / 3}\right) \gamma
\end{array}\right), \\
& G_{1}^{\Omega}=\left(\begin{array}{rrrrrrrr}
0 & 0 & 0 & 0 & 0 & 0 & 0 & 0 \\
0 & 0 & 1 & -1 & -1 & -1 & 0 & 1 \\
-1 & 0 & 0 & 1 & 0 & 1 & 0 & -1 \\
0 & -1 & 0 & -1 & 0 & -1 & 0 & 1 \\
0 & 0 & -1 & 1 & 0 & 1 & 0 & -1 \\
0 & 0 & 0 & 1 & 0 & 1 & 0 & 0 \\
0 & 0 & 0 & 0 & 0 & 0 & 0 & 0 \\
0 & 0 & 0 & 1 & 0 & 1 & 0 & -1 \\
-1 & 0 & 1 & -1 & 0 & -1 & 0 & 0 \\
0 & -1 & -1 & 0 & 0 & -1 & -1 & 0 \\
0 & 0 & 0 & 0 & 0 & 0 & 0 & 0 \\
0 & 0 & 0 & 0 & 0 & 0 & 0 & 0 \\
0 & 0 & 0 & 1 & 0 & 1 & 1 & 0 \\
0 & 0 & 0 & 0 & 0 & 0 & 0 & 0 \\
0 & 0 & 0 & 0 & 0 & 0 & 0 & 0 \\
0 & 0 & 0 & 0 & 0 & 0 & 0 & 0 \\
0 & 0 & 0 & 0 & -1 & 0 & 0 & 0 \\
0 & 0 & 0 & 0 & 0 & 0 & 0 & 0 \\
0 & 0 & 0 & 0 & 0 & 0 & 0 & 0 \\
0 & 0 & 0 & 0 & 0 & 0 & 0 & 0
\end{array}\right), \\
& \left(G_{2}^{\Omega}\right)^{T}=\left(\begin{array}{rrrrrrrr}
0 & 0 & 0 & -1 & 0 & -1 & 1 & 0 \\
0 & 0 & 1 & 0 & 0 & 0 & 0 & 1 \\
-1 & 0 & -1 & -1 & 1 & 1 & 0 & 2 \\
0 & -1 & 0 & -1 & 0 & 1 & 0 & 0 \\
0 & 0 & 1 & 0 & -1 & 0 & 0 & -1 \\
0 & 0 & 1 & 1 & -1 & 0 & 1 & -1 \\
0 & -1 & 0 & -1 & 0 & 1 & -1 & 0 \\
1 & -1 & 1 & 0 & 0 & 0 & 0 & -1 \\
0 & 0 & 1 & 1 & -1 & -1 & 0 & -2 \\
0 & 0 & 0 & 1 & 0 & -1 & 0 & 0 \\
1 & -1 & 1 & 0 & 1 & 0 & 0 & 1 \\
1 & 0 & 1 & 1 & 1 & 0 & 1 & 1 \\
0 & 0 & 0 & 1 & 0 & -1 & 1 & 0 \\
0 & 0 & -1 & 0 & 0 & 0 & 0 & 1 \\
1 & 0 & 1 & 1 & 1 & 1 & 0 & 2 \\
0 & 1 & 0 & 1 & 0 & 1 & 0 & 0 \\
0 & 0 & -1 & 0 & -1 & 0 & 0 & -1 \\
0 & 0 & -1 & -1 & -1 & 0 & -1 & -1 \\
0 & 1 & 0 & 1 & 0 & 1 & -1 & 0 \\
-1 & 1 & -1 & 0 & 0 & 0 & 0 & -1
\end{array}\right) .
\end{aligned}
$$

We have arranged these matrices so that

$$
\Omega G_{1}^{\Omega}=\left(\Omega_{8}, \Omega_{9}\right) \quad \text { and } \quad\left(\Omega_{8}, \Omega_{9}\right) G_{2}^{\Omega}=\Omega .
$$


Taking the real and imaginary parts, we get $\left(\Omega_{8}, \Omega_{9}\right)=\Omega_{\mathrm{Re}}+i \Omega_{\mathrm{Im}}$, with

$$
\begin{aligned}
& \Omega_{\mathrm{Re}}=\left(\begin{array}{ccccccccc}
3 \alpha & 3 \alpha & 0 & -\frac{3}{2} \alpha & 0 & 0 & 0 & 0 \\
0 & 0 & 0 & +\frac{3}{2} \alpha & 0 & 0 & 0 & 0 \\
0 & 0 & 0 & 0 & 6 \gamma & 0 & -\frac{3}{2} \gamma & -3 \gamma \\
0 & 0 & 0 & 0 & 0 & -\sqrt{3} \gamma & -\frac{\sqrt{3}}{2} \gamma & \sqrt{3} \gamma
\end{array}\right), \\
& \Omega_{\operatorname{Im}}=\left(\begin{array}{ccccccccc}
-\sqrt{3} \alpha & \sqrt{3} \alpha & \sqrt{3} \alpha & -\frac{\sqrt{3}}{2} \alpha & 0 & 0 & 0 & 0 \\
0 & 0 & -\sqrt{3} \alpha & +\frac{\sqrt{3}}{2} \alpha & 0 & 0 & 0 & 0 \\
0 & 0 & 0 & 0 & 0 & 0 & \frac{3 \sqrt{3}}{2} \gamma & 0 \\
0 & 0 & 0 & 0 & 0 & 3 \gamma & \frac{3}{2} \gamma & 0
\end{array}\right) .
\end{aligned}
$$

Then

$$
\Lambda:=\left(\begin{array}{cccc}
3 \alpha & \frac{3}{2} \alpha & 0 & 0 \\
0 & \frac{3}{2} \alpha & 0 & 0 \\
0 & 0 & 3 \gamma & \frac{3}{2} \gamma \\
0 & 0 & 0 & \frac{\sqrt{3}}{2} \gamma
\end{array}\right), \quad \Lambda_{\pi / 2}:=\left(\begin{array}{cccc}
\sqrt{3} \alpha & \frac{\sqrt{3}}{2} \alpha & 0 & 0 \\
0 & \frac{\sqrt{3}}{2} \alpha & 0 & 0 \\
0 & 0 & 3 \sqrt{3} \gamma & \frac{3 \sqrt{3}}{2} \gamma \\
0 & 0 & 0 & \frac{3}{2} \gamma
\end{array}\right)
$$

are related to $\Omega_{\mathrm{Re}}$ and $\Omega_{\mathrm{Im}}$ via integer matrices:

$$
\begin{array}{rlrl}
\Omega_{\mathrm{Re}} G_{1}^{R} & =\Lambda, & \Lambda G_{2}^{R}=\Omega_{\mathrm{Re}}, \\
\Omega_{\operatorname{Im}} G_{1}^{I}=\Lambda_{\pi / 2}, & \Lambda_{\pi / 2} G_{2}^{I}=\Omega_{\mathrm{Im}},
\end{array}
$$

showing that $\Omega_{\operatorname{Re}}$ spans the lattice defined by $\Lambda$ and $\Omega_{\operatorname{Im}}$ the lattice defined by $\Lambda_{\pi / 2}$. This concludes the proof that the minimal surface $f: M_{10} \rightarrow \mathbb{R}^{4} / \Lambda$ and its conjugate $f_{\pi / 2}: M_{10} \rightarrow \mathbb{R}^{4} / \Lambda_{\pi / 2}$ are well defined.

The associate surfaces. To prove the well-definedness of the associate surfaces $f_{\theta}$ of $f$, we take the period matrix of $f_{\theta}$ :

$$
\operatorname{Re}\left\{e^{i \theta} \Omega\right\}=\cos \theta\left(\Omega_{10} \quad \Omega_{11}\right)
$$


where

$$
\begin{aligned}
& \Omega_{10}=\left(\begin{array}{cccc}
(3+\sqrt{3} \tan \theta) \alpha & (3-\sqrt{3} \tan \theta) \alpha & -\sqrt{3} \alpha \tan \theta & -\frac{\alpha}{2}(3-\sqrt{3} \tan \theta) \\
0 & 0 & \sqrt{3} \alpha \tan \theta & \frac{\alpha}{2}(3-\sqrt{3} \tan \theta) \\
0 & 0 & 0 & 0 \\
0 & 0 & 0 & 0
\end{array}\right), \\
& \Omega_{11}=\left(\begin{array}{cccc}
0 & 0 & 0 & 0 \\
0 & 0 & -\frac{3 \gamma}{2}(1+\sqrt{3} \tan \theta) & -3 \gamma \\
6 \gamma & 0 & -\frac{\sqrt{3} \gamma}{2}(1+\sqrt{3} \tan \theta) & \sqrt{3} \gamma
\end{array}\right)
\end{aligned}
$$

If $\sqrt{3} \tan \theta=m / n \in \mathbb{Q}$, we can show that $\operatorname{rank}_{\mathbb{Q}} \operatorname{Re}\left\{e^{i \theta} \Omega\right\}=4$. This implies the condition Theorem 1.1(3), so each $f_{\theta}$ is well defined. Thus the associate surfaces are defined for a countable dense set of angles $e^{i \theta} \in S^{1}$ because $\theta$ is parametrized by the rationals.

\section{Homological triviality and reducible symmetry}

Finally, the proof of Main Theorem 1.5(iii) is given in the next two lemmas.

Lemma 4.1. $f\left(M_{10}\right)$ is homologous to 0 in $\mathbb{R}^{4} / \Lambda$.

Proof. Setting

$$
\left(x^{1}, x^{2}, x^{3}, x^{4}\right)=\operatorname{Re} \int_{p_{0}}^{p}\left(\frac{1-z^{6}}{w^{2}}, \frac{i\left(1+z^{6}\right)}{w^{2}}, \frac{z^{5}+z}{w^{2}}, \frac{i\left(z^{5}-z\right)}{w^{2}}\right)^{T} d z
$$

we obtain

$$
\left(\begin{array}{c}
d x^{1} \\
d x^{2} \\
d x^{3} \\
d x^{4}
\end{array}\right)=\frac{1}{2}\left(\begin{array}{c}
\frac{1-z^{6}}{w^{2}} d z+\frac{1-\bar{z}^{6}}{\bar{w}^{2}} d \bar{z} \\
\frac{i\left(1+z^{6}\right)}{w^{2}} d z-\frac{i\left(1+\bar{z}^{6}\right)}{\bar{w}^{2}} d \bar{z} \\
\frac{z^{5}+z}{w^{2}} d z+\frac{\bar{z}^{5}+\bar{z}}{\bar{w}^{2}} d \bar{z} \\
\frac{i\left(z^{5}-z\right)}{w^{2}} d z-\frac{i\left(\bar{z}^{5}-\bar{z}\right)}{\bar{w}^{2}} d \bar{z}
\end{array}\right) .
$$

It follows that 


$$
\begin{aligned}
d x^{1} \wedge d x^{2} & =\frac{1}{4}\left(\frac{1-z^{6}}{w^{2}} d z+\frac{1-\bar{z}^{6}}{\bar{w}^{2}} d \bar{z}\right) \wedge\left(\frac{i\left(1+z^{6}\right)}{w^{2}} d z-\frac{i\left(1+\bar{z}^{6}\right)}{\bar{w}^{2}} d \bar{z}\right) \\
& =-\frac{i}{2} \frac{1-|z|^{12}}{|w|^{4}} d z \wedge d \bar{z}
\end{aligned}
$$$$
d x^{1} \wedge d x^{3}=\frac{1}{4}\left(\frac{1-z^{6}}{w^{2}} d z+\frac{1-\bar{z}^{6}}{\bar{w}^{2}} d \bar{z}\right) \wedge\left(\frac{z^{5}+z}{w^{2}} d z+\frac{\bar{z}^{5}+\bar{z}}{\bar{w}^{2}} d \bar{z}\right)
$$$$
=\frac{1}{4} \frac{-(z-\bar{z})\left(1+|z|^{10}\right)-\left(z^{5}-\bar{z}^{5}\right)\left(1+|z|^{2}\right)}{|w|^{4}} d z \wedge d \bar{z},
$$$$
\begin{aligned}
d x^{1} \wedge d x^{4} & =\frac{1}{4}\left(\frac{1-z^{6}}{w^{2}} d z+\frac{1-\bar{z}^{6}}{\bar{w}^{2}} d \bar{z}\right) \wedge\left(\frac{i\left(z^{5}-z\right)}{w^{2}} d z-\frac{i\left(\bar{z}^{5}-\bar{z}\right)}{\bar{w}^{2}} d \bar{z}\right) \\
& =-\frac{i}{4} \frac{-(z+\bar{z})\left(1+|z|^{10}\right)+\left(z^{5}+\bar{z}^{5}\right)\left(1+|z|^{2}\right)}{|w|^{4}} d z \wedge d \bar{z}
\end{aligned}
$$$$
d x^{2} \wedge d x^{3}=\frac{1}{4}\left(\frac{i\left(1+z^{6}\right)}{w^{2}} d z-\frac{i\left(1+\bar{z}^{6}\right)}{\bar{w}^{2}} d \bar{z}\right) \wedge\left(\frac{z^{5}+z}{w^{2}} d z+\frac{\bar{z}^{5}+\bar{z}}{\bar{w}^{2}} d \bar{z}\right)
$$$$
=\frac{i}{4} \frac{(z+\bar{z})\left(1+|z|^{10}\right)+\left(z^{5}+\bar{z}^{5}\right)\left(1+|z|^{2}\right)}{|w|^{4}} d z \wedge d \bar{z},
$$

$$
\begin{aligned}
d x^{2} \wedge d x^{4} & =\frac{1}{4}\left(\frac{i\left(1+z^{6}\right)}{w^{2}} d z-\frac{i\left(1+\bar{z}^{6}\right)}{\bar{w}^{2}} d \bar{z}\right) \wedge\left(\frac{i\left(z^{5}-z\right)}{w^{2}} d z-\frac{i\left(\bar{z}^{5}-\bar{z}\right)}{\bar{w}^{2}} d \bar{z}\right) \\
& =\frac{1}{4} \frac{(z-\bar{z})\left(1+|z|^{10}\right)-\left(z^{5}-\bar{z}^{5}\right)\left(1+|z|^{2}\right)}{|w|^{4}} d z \wedge d \bar{z},
\end{aligned}
$$

$$
\begin{aligned}
d x^{3} \wedge d x^{4} & =\frac{1}{4}\left(\frac{z^{5}+z}{w^{2}} d z+\frac{\bar{z}^{5}+\bar{z}}{\bar{w}^{2}} d \bar{z}\right) \wedge\left(\frac{i\left(z^{5}-z\right)}{w^{2}} d z-\frac{i\left(\bar{z}^{5}-\bar{z}\right)}{\bar{w}^{2}} d \bar{z}\right) \\
& =-\frac{i}{2} \frac{-|z|^{2}+|z|^{10}}{|w|^{4}} d z \wedge d \bar{z} .
\end{aligned}
$$

Setting $z=r e^{i \theta}(0 \leq r \leq \infty, 0 \leq \theta \leq 2 \pi)$, we get $d z \wedge d \bar{z}=-2 i r d r \wedge d \theta$. First,

$$
\begin{aligned}
\int_{M_{10}} d x^{1} \wedge d x^{2} & =3 \int_{\theta=0}^{2 \pi} \int_{r=0}^{\infty} \frac{i}{2} \frac{1-r^{12}}{\sqrt[3]{\left(r^{24}-2 r^{12} \cos (12 \theta)+1\right)^{2}}} 2 i r d r d \theta \\
& =-3 \int_{\theta=0}^{2 \pi}\left(\int_{r=0}^{1}+\int_{r=1}^{\infty}\right) \\
& =-3 \int_{\theta=0}^{2 \pi}\left(\int_{r=0}^{1}-\int_{r^{\prime}=0}^{1}\right) \quad\left(r^{\prime}=1 / r\right) \\
& =0 .
\end{aligned}
$$


Similarly, we can see that $\int_{M_{10}} d x^{3} \wedge d x^{4}=0$. Next, we obtain

$$
\begin{aligned}
\int_{M_{10}} d x^{1} \wedge d x^{3} & =-3 \int_{\theta=0}^{2 \pi} \int_{r=0}^{\infty} \frac{r \sin \theta\left(1+r^{10}\right)+r^{5} \sin (5 \theta)\left(1+r^{2}\right)}{\sqrt[3]{\left(r^{24}-2 r^{12} \cos (12 \theta)+1\right)^{2}}} r d r d \theta \\
& =-3\left(\int_{\theta=0}^{\pi} \int_{r=0}^{\infty}+\int_{\theta=\pi}^{2 \pi} \int_{r=0}^{\infty}\right) \\
& =-3\left(\int_{\theta=0}^{\pi} \int_{r=0}^{\infty}-\int_{\theta^{\prime}=0}^{\pi} \int_{r=0}^{\infty}\right) \quad\left(\theta^{\prime}=\theta-\pi\right) \\
& =0 .
\end{aligned}
$$

Similarly, $\int_{M_{10}} d x^{1} \wedge d x^{4}=\int_{M_{10}} d x^{2} \wedge d x^{3}=\int_{M_{10}} d x^{2} \wedge d x^{4}=0$ follows. Therefore $\left[f\left(M_{10}\right)\right]=0$.

Lemma 4.2. The map $f$ has only reducible symmetry. The maximal symmetry of $f$ is the dihedral group $D_{12}$.

Proof. Let $S_{f}\left(M_{10}\right)$ be an arbitrary symmetry. For every $\phi \in S_{f}\left(M_{10}\right)$, we have the commutative diagram

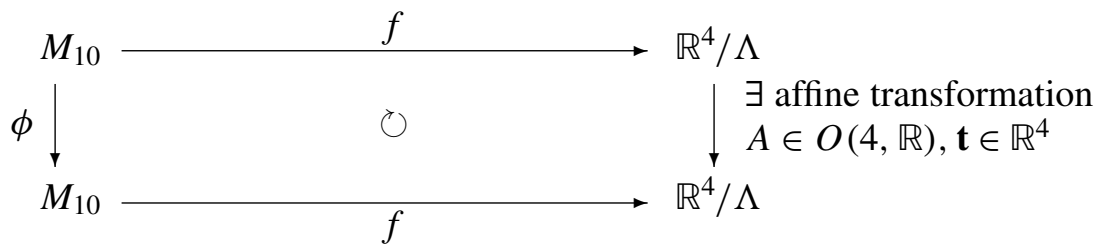

where we define the automorphism $j$ by $j(z, w)=\left(z, e^{2 \pi / 3 i} w\right)$. Because the Gauss map $G$ is $j$-invariant, $\phi$ induces an automorphism $\phi^{\prime}$ of $S^{2}$ :

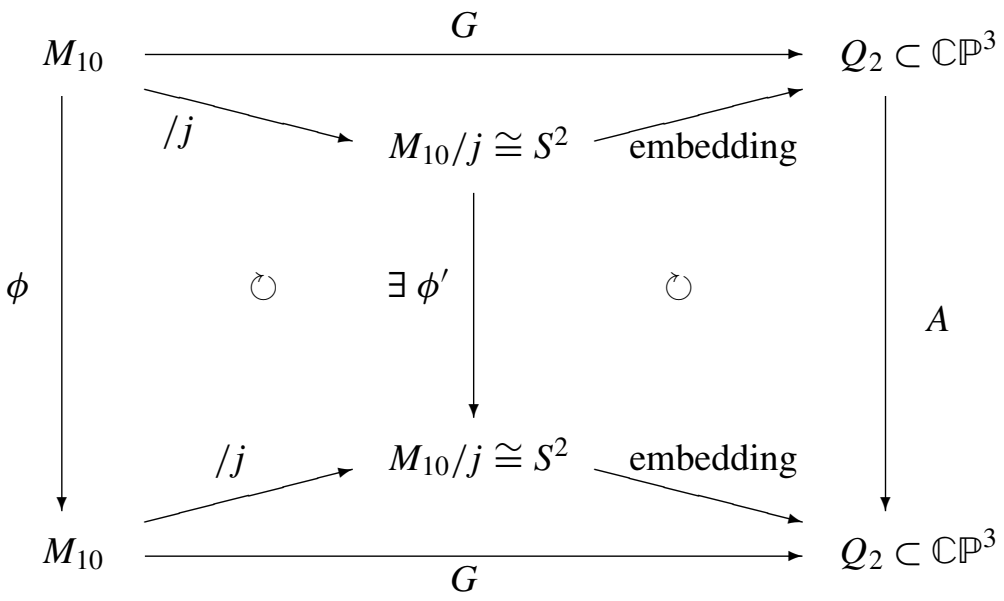

Let $S_{f}^{\prime}\left(M_{10}\right)$ be a subgroup of automorphims of $M_{10}$ which induce automorphisms of $M_{10} / j \cong S^{2} \cong \mathbb{C} \cup\{\infty\}$. Then, we obtain $S_{f}\left(M_{10}\right) \subset S_{f}^{\prime}\left(M_{10}\right)$. Now let 
$p_{\alpha}=\left(e^{(\pi / 6) \alpha i}, 0\right)(1 \leq \alpha \leq 12)$ be branch points of $/ j$. Every element of $S_{f}^{\prime}\left(M_{10}\right)$ induces an automorphism of $\mathbb{C} \cup\{\infty\}$ that preserves $\left\{e^{(\pi / 6) \alpha i}\right\}_{\alpha=1}^{12}$. It is easy to verify that a subgroup of automorphisms of $\mathbb{C} \cup\{\infty\}$ with the above property is generated by $z \mapsto e^{(\pi / 6) i} z$ and $z \mapsto 1 / z$. To lift these automorphisms, we consider the automorphism $\varphi^{\prime}$ of $M_{10}$ given by $\varphi^{\prime}(z, w)=\left(1 / z, e^{\pi i / 3} w / z^{4}\right)$. Thus $S_{f}^{\prime}\left(M_{10}\right)$ is generated by $j, \varphi$, and $\varphi^{\prime}$. Setting $\phi_{1}=\varphi \circ j^{2}$ and $\phi_{2}(z, w)=\varphi^{\prime} \circ j$, we obtain

$$
\phi_{1}^{*} f=\left(\begin{array}{cc}
R\left(\frac{\pi}{2}\right) & 0 \\
0 & R\left(-\frac{\pi}{3}\right)
\end{array}\right) f, \quad \phi_{2}^{*} f=\left(\begin{array}{rrrr}
1 & 0 & 0 & 0 \\
0 & -1 & 0 & 0 \\
0 & 0 & -1 & 0 \\
0 & 0 & 0 & 1
\end{array}\right) f .
$$

Obviously, $\phi_{1}$ and $\phi_{2}$ are reducible actions respectively and generate the dihedral group $D_{12}$. Note that $j$ does not induce any affine transformation of the torus. Hence, $S_{f}\left(M_{10}\right)$ is a subgroup of $D_{12}$ generated by $\phi_{1}$ and $\phi_{2}$. Therefore, $S_{f}\left(M_{10}\right)$ has only reducible symmetry and its maximal symmetry is $D_{12}$, generated by $\phi_{1}$ and $\phi_{2}$.

\section{References}

[Arbarello et al. 1985] E. Arbarello, M. Cornalba, P. A. Griffiths, and J. Harris, Geometry of algebraic curves, vol. I, Grundlehren der Math. Wissenschaften 267, Springer, New York, 1985. MR 86h:14019 Zbl 1061.92066

[Arezzo and Pirola 1999] C. Arezzo and G. P. Pirola, "On the existence of periodic minimal surfaces”, J. Algebraic Geom. 8:4 (1999), 765-785. MR 2000f:53086 Zbl 0966.53009

[Coppens and Martens 2000] M. Coppens and G. Martens, "Linear series on 4-gonal curves", Math. Nachr. 213 (2000), 35-55. MR 2001h:14037 Zbl 0972.14021

[Coppens et al. 1992] M. Coppens, C. Keem, and G. Martens, "Primitive linear series on curves", Manuscripta Math. 77:2-3 (1992), 237-264. MR 93j:14028 Zbl 0786.14016

[Ejiri 2002] N. Ejiri, “A differential-geometric Schottky problem, and minimal surfaces in tori”, pp. 101-144 in Differential geometry and integrable systems (Tokyo, 2000), edited by M. Guest et al., Contemp. Math. 308, Amer. Math. Soc., Providence, RI, 2002. MR 2004b:58015 Zbl 1071.58012

[Hoffman and Osserman 1980] D. A. Hoffman and R. Osserman, The geometry of the generalized Gauss map, Mem. Amer. Math. Soc. 236, Amer. Math. Soc., Providence, RI, 1980. MR 82b:53012 Zbl 0469.53004

[Karcher 1989] H. Karcher, "The triply periodic minimal surfaces of Alan Schoen and their constant mean curvature companions”, Manuscripta Math. 64:3 (1989), 291-357. MR 90g:53010 Zbl 0687.53010

[Kusner and Schmitt 1995] R. Kusner and N. Schmitt, "The spinor representation of minimal surfaces", preprint, 1995. math.DG/9512003

[Meeks 1990] W. H. Meeks, “The theory of triply periodic minimal surfaces”, Indiana Univ. Math. J. 39:3 (1990), 877-936. MR 92e:53012 Zbl 0721.53057

[Miranda 1995] R. Miranda, Algebraic curves and Riemann surfaces, Graduate Studies in Mathematics 5, American Mathematical Society, Providence, RI, 1995. MR 96f:14029 Zbl 0820.14022 
[Nagano and Smyth 1976] T. Nagano and B. Smyth, "Minimal surfaces in tori by Weyl groups", Proc. Amer. Math. Soc. 61:1 (1976), 102-104. MR 55 \#4049 Zbl 0357.53005

[Nagano and Smyth 1980] T. Nagano and B. Smyth, "Periodic minimal surfaces and Weyl groups", Acta Math. 145:1-2 (1980), 1-27. MR 84c:53054 Zbl 0449.53042

[Schoen 1970] A. Schoen, "Infinite periodic minimal surfaces without self-intersections", Technical Note D-5541, NASA Electronics Res. Center, Cambridge, MA, 1970, Available at ntrs.nasa.gov/ archive/nasa/casi.ntrs.nasa.gov/19700020472_1970020472.pdf.

[Shoda 2004] T. Shoda, "New components of the moduli space of minimal surfaces in 4-dimensional flat tori”, J. London Math. Soc. (2) 70:3 (2004), 797-816. MR 2005g:53109 Zbl 1085.53050

[Shokurov and Danilov 1994] V. V. Shokurov and V. L. Danilov, Algebraic geometry, I: Algebraic curves. Algebraic manifolds and schemes, Encyclopaedia of Mathematical Sciences 23, Springer, Berlin, 1994. MR 95b:14001

Received March 22, 2006. Revised February 26, 2007.

TOSHIHIRO SHODA

FACUlTy OF CULTURE AND EDUCATION

SAGA UNIVERSITY

1 HONJO-CHO

SAGA-CITY, SAGA, 840-8502

JAPAN

tshoda@cc.saga-u.ac.jp 